\title{
Correction to: Extent and Location of the Excitatory and Inhibitory Cortical Hand Representation Maps: A Navigated Transcranial Magnetic Stimulation Study
}

\author{
Minna Pitkänen $^{1,2} \cdot$ Elisa Kallioniemi $^{1,3} \cdot$ Petro Julkunen $^{1,3}$
}

Published online: 14 October 2017

(C) Springer Science+Business Media, LLC 2017

\section{Correction to: Brain Topogr (2015) 28:657-665 DOI 10.1007/s10548-015-0442-6}

The original version of this article unfortunately contained an error. An error in the transformation between coordinate systems used to derive part of the results has been noticed.

The error affected only the results of the center of gravities (CoGs) in the anterior-posterior direction, while other data was correctly analyzed and reported. We have reanalyzed part of the data with corrected coordinate values. This has led to some changes in the originally reported results.

Contrary to what was reported in the original article, the muscle of interest did not influence the location of the motor evoked potential (MEP) or silent period (SP) CoGs in anteriorposterior direction $(p=0.100)$. The mean difference between MEP and SP CoGs in the anterior-posterior direction was $0.8 \mathrm{~mm}$ instead of the reported $0.6 \mathrm{~mm}$. Although, not affecting the conclusions, we have corrected the reported statistics values in the results:

"MEP CoGs were more medial $(p<0.001)$ and anterior $(p=0.001)$ than the SP CoGs on the dominant hemisphere."

The online version of the original article can be found under doi:10.1007/s 10548-015-0442-6.

Minna Pitkänen

minna.pitk@gmail.com

1 Department of Clinical Neurophysiology, Kuopio University Hospital, POB 100, 70029 KYS, Finland

2 Department of Neuroscience and Biomedical Engineering, Aalto University School of Science, POB 12200, 00076 Aalto, Finland

3 Department of Applied Physics, University of Eastern Finland, POB 1627, 70211 Kuopio, Finland
"The SP CoGs were more anterior on the non-dominant hemisphere than on the dominant side $(p<0.001)$."

"The grid did not affect the CoGs in anterior-posterior direction $(F=0.06, p=0.807)$."

The error resulted in erroneous Fig. 3, which has now been corrected to show the differences between the MEP and SP CoGs.

Other reported results were not influenced by the error and the conclusions remain the same with the exception that the representations of different muscles differed only based on size and not based on the CoGs. 

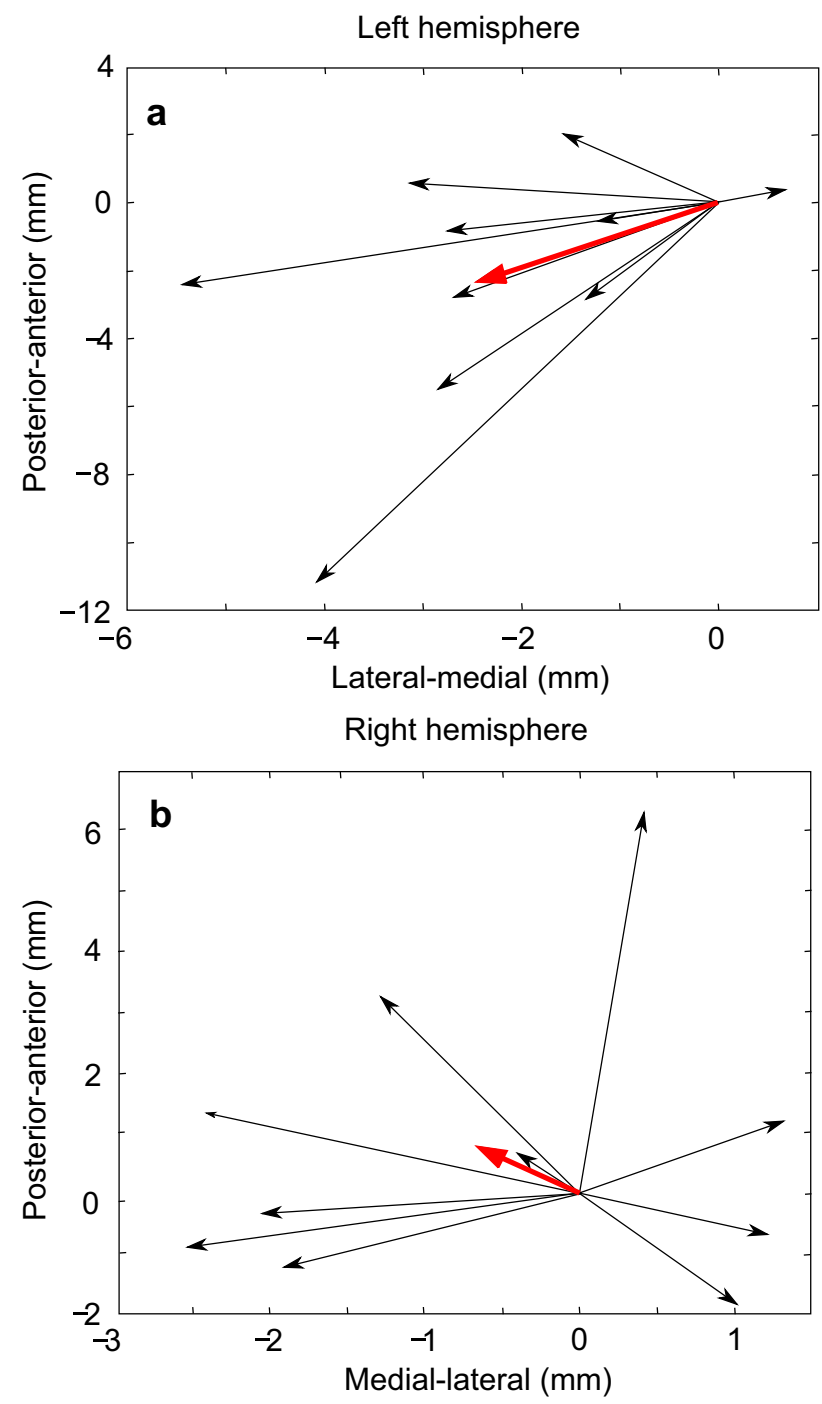

Fig. 3 The distances between the MEP and SP CoGs for each subject in the left (a) and right (b) hemisphere. The MEP CoGs are centered at the origin and the arrows point from the MEP CoGs to the SP CoGs. The means are represented as red bolded arrows 